\title{
Suicide in Parkinson' s Disease: An Open Question and a Complex and Poorly Explored Phenomenon
}

\author{
Antonino Cannas ${ }^{*}$, Mario Meloni* and Francesco Marrosu \\ Department of Neurology, Movement Disorders Center, Policlinico Universitario Monserrato, University of Cagliari, Italy
}

*Corresponding author: Mario Meloni, Department of Neurology, Movement Disorders Center, Policlinico Universitario Monserrato, University of Cagliari, SS 554 Bivio per Sestu, 09042 Monserrato, Cagliari, Italy, Tel: +39 0705109 6001; E-mail: mario.meloni@hotmail.it

Rec date: May 12, 2016; Acc date: Jun 29, 2016; Pub date: June 30, 2016

Copyright: ( 2016 Cannas A, et al. This is an open-access article distributed under the terms of the Creative Commons Attribution License, which permits unrestricted use, distribution, and reproduction in any medium, provided the original author and source are credited.

Keywords: Suicide; Suicidal ideation; Parkinson's disease; Deep brain stimulation; Levodopa carbidopa intestinal gel; Dopamine agonist withdrawal syndrome

\section{Letter to Editor}

Susceptibility to suicide and suicidal ideation is a very serious issue in patients with Parkinson's disease (PD). There is much that is still unknown about the relationship between suicide, age, medical treatment and disease in the PD patient population. Depression is the most common psychiatric disturbance that affects people with $\mathrm{PD}$. On multivariate analysis, severity of depression and psychosis were the only predictors of suicide or death ideation for PD patients $[1,2]$. Recent reports of an increased suicide risk associated with subthalamic nucleus deep-brain stimulation (DBS) and levodopa-carbidopa intestinal gel (LCIG) in advanced PD call attention to the need for systematic investigation of risk factors and psychopathological basis related to suicidal and death ideation [3-7]. While it is now recognized that DBS may increase the risk of suicide, probably through increased impulsiveness, 3 the data for LCIG are still emerging [5-7]. The possible precipitating role of the dopamine agonist withdrawal syndrome (DAWS) in PD patients under LCIG and previously treated with dopamine agonist is gaining attention in the scientific literature [8]. However, the pathophysiology of suicide is extremely complex and evidence of DAWS being directly or indirectly associated with suicidal behavior remains meagre.

We report a recent case of suicide in a patient under LCIG therapy. The patient was a 75-year-old male with a 15 -year history of PD. He had an advanced PD (III-IV of Hoehn/Yahr score) with mild cognitive impairment $(\mathrm{MMSE}=26)$. At the psychiatric evaluation emerged only a mild dysthymia which not required specific antidepressant treatments. Two years before starting LCIG, the patient experienced an isolated episode of short-term visual hallucinations clearly related to the introduction of pramipexole. During follow-up visits, there were no signs of impulse control disorders or psychosis but substantial decrease of motor function and unpredictable levodopa induced motor fluctuations. Unified Parkinson Disease Rating Scale motor subsection part III (UPDRS III) scores during off state was 60 of 108, whereas UPDRS III while on state was 28 of 108 . At that time, he was taking immediate-release levodopa/carbidopa at the dose of $100 / 25 \mathrm{mg}, 5$ times daily and controlled-release levodopa/benserazide at the dose of 100/25 mg, 3 times daily. Treatment with LCIG was proposed in order to obtain a lower and more stable therapeutic regimen. LCIG infusion was started in the morning after awakening and stopped before bedtime. A significant reduction of the motor fluctuations were obtained through a LCIG titration of the morning dose to $10 \mathrm{ml}(200$ $\mathrm{mg}$ of levodopa), of the continuous dose to $1.9 \mathrm{ml}$ (38 $\mathrm{mg}$ ) per hour per $16 \mathrm{hrs}$ a day (total dose of $808 \mathrm{mg}$ ) and of the extra-doses to $1.5 \mathrm{ml}$
(30 mg). During follow-up evaluations we observed marked improvement in the UPDRS motor subscale scores without reporting any neuropsychiatric adverse effects, such as confusion, psychosis, depression or mania. Notably, suicidal ideation was not observed. One year before death, he referred only mild insomnia and quetiapine (25 $\mathrm{mg}$ at night) was started. At the last follow-up observation, he had no evident signs of depression and feeling relaxed with adequate sleep was reported. The patient committed suicide by hanging from a tree, eighteen months after LCIG initiation and 1 month since the last follow-up visit. LCIG and DBS are available options for patients at advanced stages of the disease. Case reports and observational studies, with long-term follow-up, have suggested that DBS lead to suicide attempts or completed suicide in a subset of PD patients. [3,4,9] In patients treated with DBS, suicidal behaviour was associated with postoperative depression and/or altered impulse regulation 3 . Although an increase of suicidal behaviors in PD patients has been described in clinical trials involving DBS 3, the data for LCIG are still emerging [5-7]. Our patient experienced a single episode of hallucinations induced by pramipexole. His psychosis recovered gradually after discontinuation of dopamine agonist therapy. There was a fairly good familiar support, and suicidal ideation or attempts before LCIG therapy were not reported. In our case, DAWS per se must not be considered as the cause of suicidal ideation and other causative factors must be considered. The "hypodopaminergic syndrome" caused by dopamine agonist withdrawal is associated with depression, anxiety, apathy and anhedonia. We argue that this constellation of symptoms may not be sufficient to cause a suicide attempt. In fact, the patient committed suicide (despite excellent motor improvements reported under LCIG therapy) by hanging himself on a two meters high tree in a rural area located two km away from his home. In our opinion, this is difficult to realize in a condition of apathetic and anhedonic state. Recent studies report a broad range of rates of attempted $(1.6 \%$ to $4.3 \%)[1,10]$ or completed suicides $(0.8 \%$ to $2 \%)[2,10]$ in medically treated groups. In our experience, suicide may be more common in LCIG-treated patients, because we have witnessed 1 (4\%) case of suicide among 25 PD patients treated with LCIG from 2012 to 2015 and no suicide observation in 20 years with the traditional oral therapy. We speculated that an explanation of the increase in suicide rate should be sought in a different pattern of dopaminergic stimulation occurred after the LCIG era. Gradual reduction or withdrawal of dopaminergic drugs within a context of a personalized pharmacological management is the same today as it was before the pre-DBS/LCIG era. However, the suicide rate has increased since the introduction of these new therapeutic strategies. We argue that the improvement in motor performance with effective treatments (DBS as well as LCIG) is not always followed by a sufficient restoring of an affective and emotional reward process, so this condition might become a possible cause to attempt or commit suicide. A good "on" 
Citation: Cannas A, Meloni M, Marrosu F (2016) Suicide in Parkinson's Disease: An Open Question and a Complex and Poorly Explored Phenomenon. J Neurol Disord 4: 273. doi:10.4172/2329-6895.1000273

Page 2 of 2

motor state is not necessarily followed by an optimal "on" affective state and this could be one of the causes of the execution of preexisting suicidal thoughts.

\section{References}

1. Nazem S, Siderowf AD, Duda JE, Brown GK, Ten Have T, et al. (2008) Suicidal and death ideation in Parkinson's disease. Mov Disord 23: 1573-1579.

2. Kostic VS, Pekmezovic T, Tomic A, Jecmenica-Lukic M, Stojkovic T, et al. (2010) Suicide and suicidal ideation in Parkinson's disease. J Neurol Sci 289: 40-43.

3. Voon V, Krack P, Lang AE, Lozano AM, Dujardin K, et al. (2008) A multicentre study on suicide outcomes following subthalamic stimulation for Parkinson's disease. Brain 131: 2720-2728.

4. Rodrigues AM, Rosas MJ, Gago MF, Sousa C, Fonseca R, et al. (2010) Suicide attempts after subthalamic nucleus stimulation for Parkinson's disease. Eur Neurol 63: 176-179.

5. Santos-García D, Macías M, Llaneza M, Aneiros A (2009) Suicide following duodenal levodopa infusion for Parkinson's disease. Mov Disord 24: 2029-2030.
6. Fernandez HH, Standaert DG, Hauser RA, Lang AE, Fung VS, et al. (2015) Levodopa-carbidopa intestinal gel in advanced Parkinson's disease: final 12-month, open-label results. Mov Disord 30: 500-509.

7. Zorko N, Kojovic M, Flisar D, Pirtosek Z, Kramberger MG (2015) Suicide in Parkinson's disease patients treated with levodopa-carbidopa intestinal gel. Mov Disord 30: 1434-1435.

8. Solla P, Fasano A, Cannas A, Marrosu F (2015) Suicide and dopamine agonist withdrawal syndrome in Parkinson's disease. Mov Disord 30: 1859-1860.

9. Funkiewiez A, Ardouin C, Caputo E, Krack P, Fraix V, et al. (2004) Long term effects of bilateral subthalamic nucleus stimulation on cognitive function, mood, and behaviour in Parkinson's disease. J Neurol Neurosurg Psychiatry 75: 834-839.

10. Schuepbach WM, Rau J, Knudsen K, Volkmann J, Krack P, et al. (2013) Neurostimulation for Parkinson's disease with early motor complications. N Engl J Med 368: 610-622. 